\title{
Acute Clenbuterol Overdose Resulting in Supraventricular Tachycardia and Atrial Fibrillation
}

\author{
G. Patrick Dauberta, Vincent H. Mabasa ${ }^{b}$, Vivian WY Leung ${ }^{b}$, Cynthia Aaron ${ }^{c, d}$
}

aDepartment of Emergency Medicine, University of California, Davis, Medical Center, Sacramento, CA

bFaculty of Pharmaceutical Sciences, University of British Columbia, Vancouver, British Columbia, Canada

cDepartment of Emergency Medicine \& Pediatrics, Wayne State University School of Medicine, Detroit, MI

dChildren's Hospital of Michigan Regional Poison Control Center, Detroit, MI

\section{ABSTRACT}

Objective: We are presenting a case illustrating the complex metabolic and rhythm disturbances associated with acute clenbuterol intoxication.

Background: Clenbuterol is a long-acting $\beta_{2}$-adrenergic agonist primarily used in veterinary medicine in the United States. It has become a common drug of abuse by body builders because of its reported anabolic and lipolytic properties. In this case report, a body builder using veterinary clenbuterol developed significant electrolyte and cardiac manifestations.

Case Report: A 31-year-old man presented to the emergency department approximately 30 minutes after ingesting $1.5 \mathrm{ml}$ (a tenfold dosing error) of Ventipulmin ${ }^{\circledR}$ syrup $(72.5 \mathrm{mcg} / \mathrm{ml}$ clenbuterol $\mathrm{HCl})$. The product was brought to the emergency department (ED) by the patient. He reported no current use of anabolic steroids. He presented in an anxious state with complaints of palpitations and shortness of breath. Vital signs upon examination were as follows: BP, 122/77 mmHg (16.3/10.3 kPa); HR 254 bpm; RR, $22 \mathrm{bpm}$; Temperature, $97.1^{\circ} \mathrm{F}\left(36^{\circ} \mathrm{C}\right)$; and oxygen saturation, $100 \%$ on ambient air. His electrocardiogram (ECG) demonstrated supraventricular tachycardia with a ventricular rate of $254 \mathrm{bpm}$. Esmolol was recommended for rate control after the unsuccessful use of adenosine and diltiazem. Laboratory studies showed potassium, $2.1 \mathrm{mmol} / \mathrm{L} ;$ magnesium, $1.3 \mathrm{mg} / \mathrm{dL}(0.54 \mathrm{mmol} / \mathrm{L}) ; \mathrm{phosphorus,} 1.0 \mathrm{mg} / \mathrm{dL}$

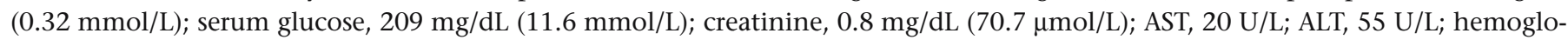
bin, $12.6 \mathrm{~g} / \mathrm{dL}(126 \mathrm{~g} / \mathrm{L})$; CPK total, $87 \mathrm{U} / \mathrm{L}$; and troponin I, $0.23 \mu \mathrm{g} / \mathrm{L}$. The patient's urine was negative for any drugs of abuse. Clenbuterol levels were not obtained. A second ECG, 16 hours post ingestion, reflected atrial fibrillation with a ventricular rate of 125 to $147 \mathrm{bpm}$. On hospital day 3, he was electively cardioverted to sinus rhythm; heart rate and rhythm returned to normal, and he was discharged with oral metoprolol.

Discussion: Clenbuterol is approved for use in countries outside the U.S. as a bronchodilator for the treatment of acute asthma exacerbations in humans. Although clenbuterol is not a steroid hormone, it possesses anabolic properties that increase muscle mass. Its longer duration of action compared to other $\beta_{2}$-agonists (such as albuterol) make it a desired agent for body-building because of its high and prolonged serum level. The mechanism for the short and long-term cardiovascular complications of clenbuterol is complex. The anabolic effects of clenbuterol are associated with its $\beta_{2}$-adrenoreceptor agonist activity on striated skeletal muscles. In addition, clenbuterol promotes lipolysis through adipocyte $\beta_{3}$-adrenoreceptors.

Conclusion: Considering the significant number of body-building enthusiasts, physicians will continue to encounter clenbuterol abuse in their clinical practices.

Keywords: clenbuterol, body-builder, intoxication, atrial fibrillation Notes: There was no outside funding off any kind used for this study.

Corresponding Author: G. Patrick Daubert, Department of Emergency Medicine, University of California, Davis, Medical Center, 4150 V Street, PSSB 2100, Sacramento, CA 95817. Email: Patrick.daubert@ucdmc.ucdavis.edu 


\section{INTRODUCTION}

Medications and chemicals used by body-building enthusiasts continue to mystify the clinician. Clenbuterol hydrochloride, a $\beta_{2}$-adrenoreceptor agonist used for the treatment of asthma and chronic obstructive pulmonary disease (COPD), is quickly gaining popularity because of its anabolic effects and ability to promote lipolysis [1-6]. However, misuse from inexperienced users have resulted in severe adverse reactions [1,7-11]. We are reporting the case of a previously healthy 31-year-old man who mistakenly took an overdose of clenbuterol resulting in supraventricular tachycardia and atrial fibrillation that required cardioversion.

\section{CASE REPORT}

A 31-year-old man presented to the emergency department approximately 30 minutes after ingesting $1.5 \mathrm{ml}$ of Ventipulmin ${ }^{\circledR}$ syrup $(72.5 \mathrm{mcg} / \mathrm{ml}$ clenbuterol $\mathrm{HCl})$. The patient acquired the medication from a veterinary medicinal supply source on the Internet. His other medications included tamoxifen, citrate flax seed oil, taurine, and multivitamin supplements.

He was anxious and complained of palpitations and shortness of breath. There were no visible signs of steroid use other than his general physique. Vital signs upon presentation were BP, 122/77 mmHg (16.3/10.3 kPa); HR, 254 bpm; RR, 22 bpm; Temperature, $97.1^{\circ} \mathrm{F}\left(36^{\circ} \mathrm{C}\right)$; and oxygen saturation, $100 \%$ on ambient air. The ECG demonstrated a supraventricular tachycardia with a ventricular rate of $254 \mathrm{bpm}$ (Figure 1). Adenosine was intravenously administered at doses of $6 \mathrm{mg}$ and $12 \mathrm{mg}$ without affect. A $10 \mathrm{mg}$ IV bolus of diltiazem decreased heart rate to $150 \mathrm{bpm}$. The patient continued to complain of mild palpitations and dyspnea, but his feelings of anxiety improved. At this point, the Poison Center was contacted and the toxicologist on call recommended esmolol as a primary agent. Sixteen hours post ingestion, the patient's heart rate continued to improve. However, repeat examination and ECG reflected atrial fibrillation with a ventricular rate of 125 to $147 \mathrm{bpm}$ (Figure 2). Laboratory studies on admission were remarkable for potassium, $2.1 \mathrm{mmol} / \mathrm{L}$; magnesium, $1.3 \mathrm{mg} / \mathrm{dL}$ (0.54 mmol/L), phosphorus, $1.0 \mathrm{mg} / \mathrm{dL}(0.32 \mathrm{mmol} / \mathrm{L})$; serum glucose, $209 \mathrm{mg} / \mathrm{dL}$ (11.6 mmol/L); creatinine, $0.8 \mathrm{mg} / \mathrm{dL}$ (70.7 $\mu \mathrm{mol} / \mathrm{L}) ;$ AST, $20 \mathrm{U} / \mathrm{L} ;$ ALT, $55 \mathrm{U} / \mathrm{L}$; hemoglobin, $12.6 \mathrm{~g} / \mathrm{dL}$ (126 g/L); CPK total, $87 \mathrm{U} / \mathrm{L}$; and troponin I, $0.23 \mu \mathrm{g} / \mathrm{L}$. The patient's urine was negative for amphetamines, barbiturates, benzodiazepines, THC, cocaine, or opiates (Dimension ${ }^{\circledR}$ Xpand ${ }^{\circledR}$ Plus System, Dade Behring, 2005). Clenbuterol levels were not obtained since the product was available and his clinical presentation was consistent with acute clenbuterol exposure. An echocardiogram revealed normal left ventricular function with an estimated ejection fraction of 55-60\%. After 48 hours of continual atrial fibrillation, he was electively cardioverted to sinus rhythm on hospital day three. The patient was started on oral metoprolol and discharged on hospital day 4; heart rate and sinus rhythm were normal, and he left without active complaints. He was advised not to use clenbuterol as part of his body-building regimen.

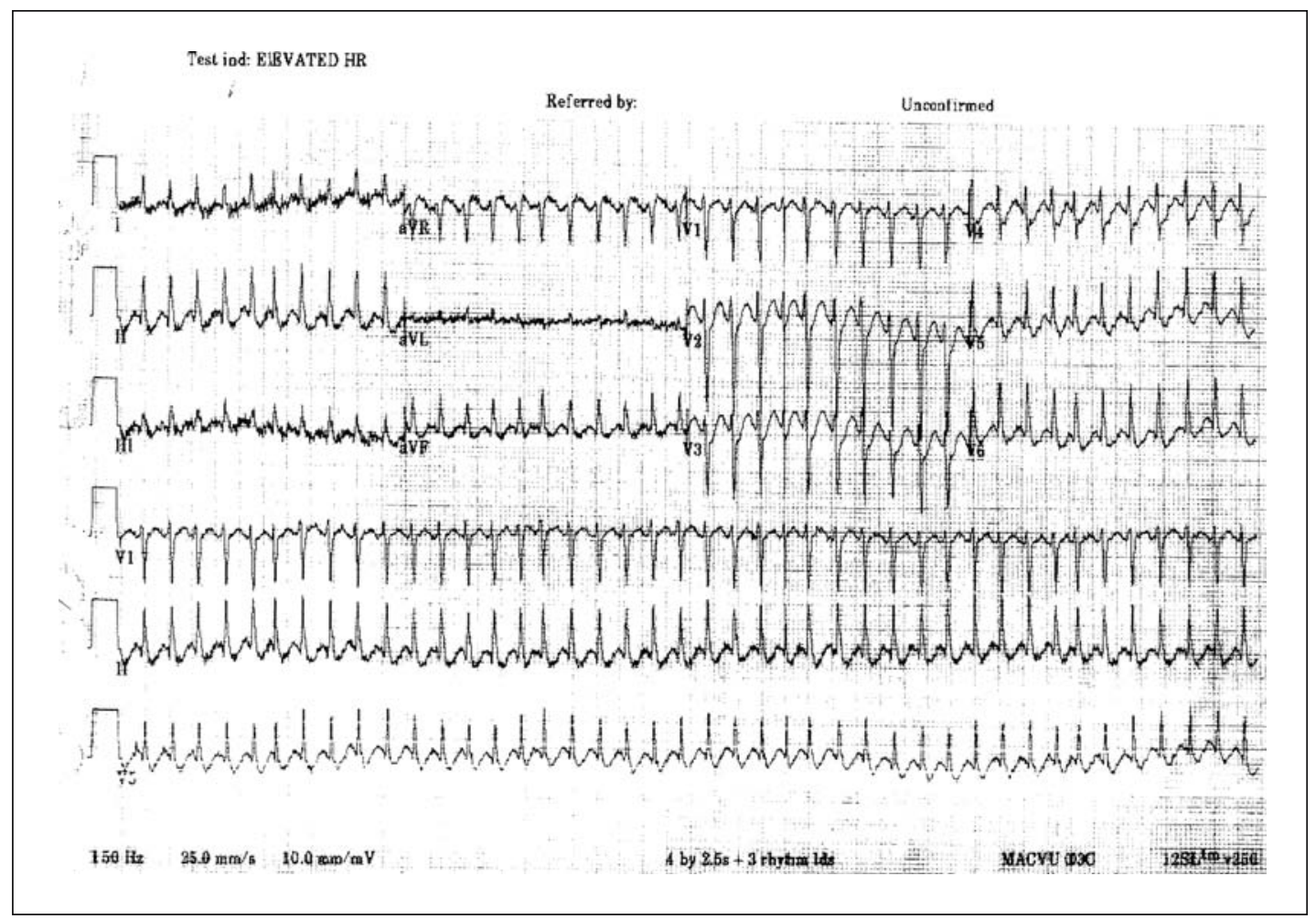

Figure 1 


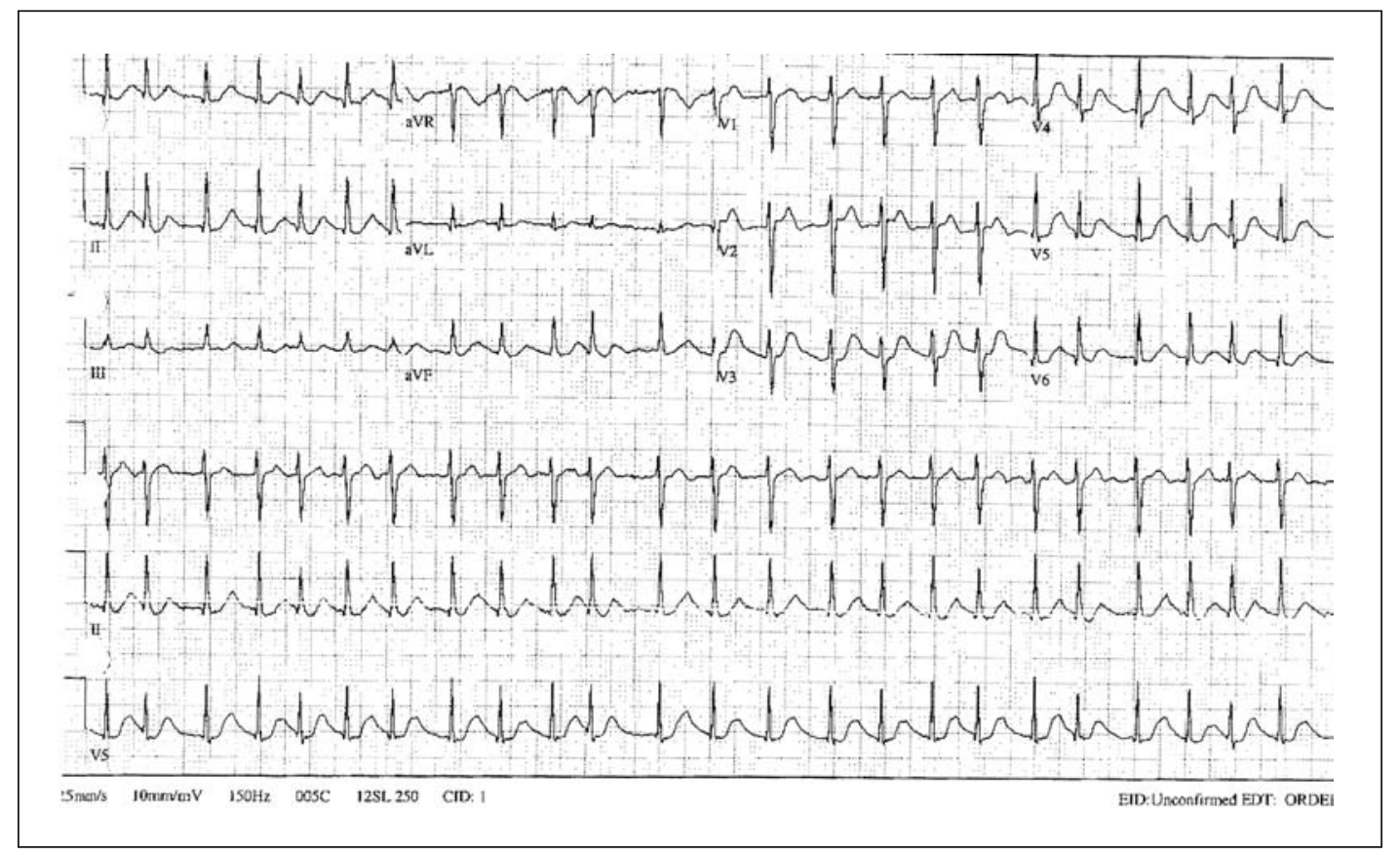

Figure 2

\section{DISCUSSION}

Outside of the United States, clenbuterol hydrochloride (Figure 3) is approved for human use as a bronchodilator in the treatment of acute asthma exacerbations. It is US approved as a veterinary product for horses experiencing respiratory diseases and for cows as a uterine relaxant at parturition [8]. As an investigational human pharmaceutical, clenbuterol has been studied in lower extremity rehabilitation and in other disease states (such cachexia and sepsis) that require muscle regeneration $[5,6]$. Although clenbuterol is not a steroid hormone, it possesses anabolic properties that athletes use to increase muscle mass. Athletes use it in combination with other performance-enhancing products (such as growth hormones and steroids) to counter their catabolic properties and to enhance muscle size [2]. In the United States there are approximately 1 million users of anabolic steroids annually; of these users, a growing number now use clenbuterol in their body-building regimen $[1,12,13]$.

Clenbuterol is available as syrup, tablets, injectable solutions, and aerosols. It is sold in Austria, Germany, Italy, Mexico, and Spain as a prescription drug for the treatment of acute asthma exacerbations. In the United States, the FDA approved clenbuterol for the management of horses affected with airway obstruction; it is not intended for human use or for livestock feeds [9]. However, it is illegally sold in health food stores, by mail-order pharmacies, and on the Internet as a nutritional supplement [2]. Clenbuterol is relatively inexpensive. One source lists the costs at $\$ 1.20$ per $0.02 \mathrm{mg}$ tablet [14]. Various $\beta_{2}$-agonists (such as clenbuterol) are banned by athletic organizations, including the United States Olympic Committee $[2,6]$. Only the

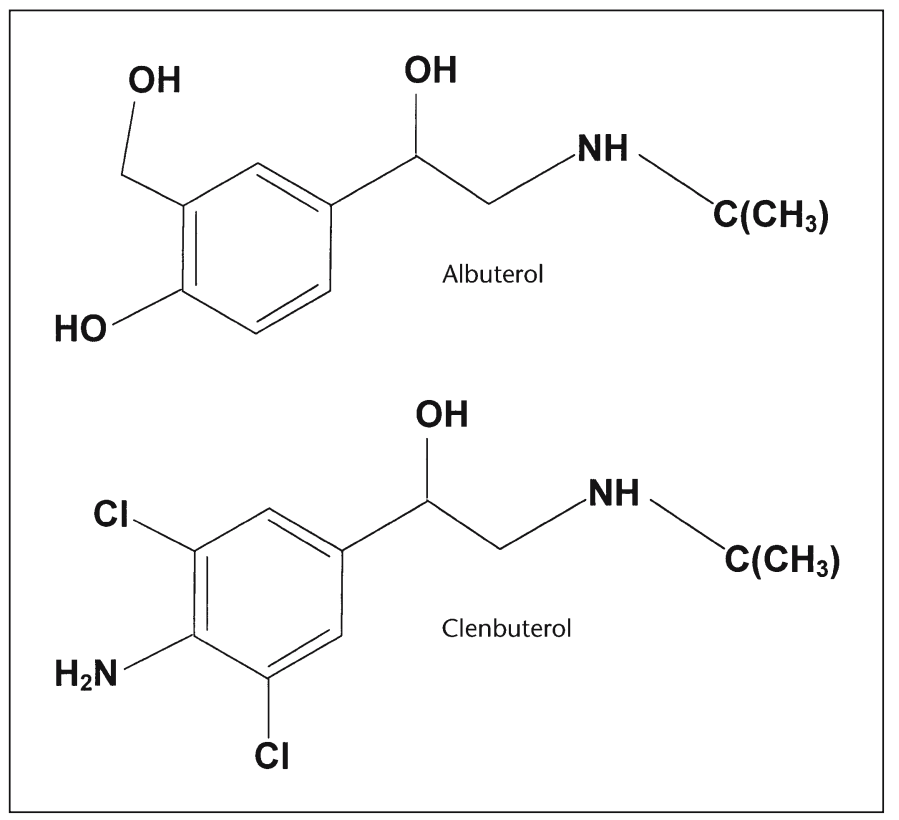

Figure 3

inhaled dosage forms of albuterol or terbutaline are allowed in competition [6].

Compared to other $\beta_{2}$-agonists, the pharmacokinetic properties of clenbuterol are unique. It is well absorbed orally with a bioavailability of $70 \%$ to $80 \%$. It has a prolonged elimination half-life of 25 to 39 hours [10]. Bronchodilation generally peaks within 3 hours, but it can persist for up to 12 hours following oral dosing. In comparison, albuterol has a significant hepatic 
first-pass metabolism, yielding 50\% bioavailability and an elimination half-life of 5-6 hours. Approximately 30\% of an oral dose of clenbuterol is largely and rapidly excreted in the urine; the majority of the remaining dose is excreted in the faeces $[10,15]$. Clenbuterol is hepatically metabolized, and five inactive metabolites have been identified [15]. The recommended clenbuterol dose for people with asthma is 20 to 40 micrograms orally twice daily, or $20 \mu \mathrm{g}$ by inhalation given at 8 -hour intervals. The initial equine dose is 0.8 micrograms/kg orally twice daily [16]. There are no standardized clenbuterol dosing regimens for body-building, and so recommendations are usually based on the anecdotal experiences of illicit users [10]. It should be noted that commonly used doses are as high as $200 \mu$ g orally taken 1 to 3 times daily over 6 to 12 week cycles $[2,11]$.

Clenbuterol's anabolic effects are associated with the $\beta_{2}$ adrenoreceptor agonist activity on striated skeletal muscles. Its longer duration of action yields a prolonged high serum level as compared to other $\beta_{2}$-agonists: this makes clenbuterol a desired agent for body-building $[4,5]$. Clenbuterol increases the rate of muscle protein deposition leading to enlargement of individual muscle fibers $[2,5,6]$. In addition, clenbuterol promotes lipolysis in humans and animals through adipocyte $\beta_{3}$-adrenoreceptors, encouraging the body to burn fatty acids [4-6]. Therefore, weight gain is a result of muscle hypertrophy and not fat accumulation. These beneficial properties make clenbuterol a highly desired ergogenic product of body-builders.

Using clenbuterol for increasing muscle mass places users at a high risk of experiencing adverse reactions because the dosages used are greater than those commonly recommended for the treatment of respiratory disease. Although clenbuterol is specific for the $\beta_{2}$-adrenoreceptor, at high doses it will act as a $\beta_{1}$-agonist. The dose-dependent $\beta$-agonist-related adverse effects include headache, dizziness, nervousness, mild tachycardia, palpitations, hypokalemia, hyperglycemia, nausea, muscle tremors, tenseness, and peripheral vasodilation $[2,8,9]$. Excessive ingestions can lead to hallucinations, severe headaches, convulsions, nausea, and vomiting $[17,18]$.

The mechanism for short and long term cardiovascular complications is complex. Hypotension, severe chest pain, tachycardia, dysrhythmias, and myocardial ischemia are various adverse cardiac events reported with clenbuterol use [1]. There are case reports of ventricular hypertrophy and tachycardia, myocardial infarction, and sudden death after the use of clenbuterol-either alone or combined with anabolic steroids $[1,2,11]$. The anabolic properties of clenbuterol probably affect the cardiac myocyte similar to anabolic steroids $[10,11]$. Although lone atrial fibrillation, due to clenbuterol, has been reported in other exposures, this is the first described case of atrial fibrillation in a body-builder using clenbuterol $[19,22]$. The incidence of atrial fibrillation from more typical $\beta_{2}$-adrenoreceptor agonists (albuterol) is equally uncommon. The role of the $\beta_{2}$-adrenergic receptor in dysrhythmias is of interest due to the wide-spread use of these medications in patients with asthma and COPD. $\beta_{1}$ - adrenergic receptors and ${ }_{2}$-adrenergic receptors coexist in the heart, generally in a ratio of
$3: 1$, respectively [23]. $\beta_{2}$-adrenergic receptors are also present on adrenergic nerve terminals in the heart where they facilitate norepinephrine release [23]. It is well known that $\beta_{2}$-adrenoreceptormediated chronotropic and inotropic responses contribute to the cardiac responses from $ß$-agonists. Compensatory cardiac hypertrophy, resulting from an increase in hemodynamic demand from enhanced muscle growth, increases the risk of arrhythmias [11]. The stimulation of either beta receptor results in positive inotropic and chronotropic responses, cardiac myocyte growth, and cardiac toxicity [23]. The majority of $\beta_{2}$-adrenoceptor agonists have an intermediate efficacy dependent upon receptor density and coupling [24]. The sole effect of $\beta_{2}$-adrenoceptor agonists on cardiac myocytes is not well studied. However, receptor characteristics of drug affinity and drug efficacy in bronchial smooth muscle are well described in the literature. Using isoproterenol as an example of a drug with a high efficacy in the bronchial smooth muscle, albuterol and terbutaline are of moderate efficacy (65-85\%), and clenbuterol is of low efficacy (40\%) [24]. Low efficacy in a $ß_{2}$-adrenoceptor agonist does not compromise its clinical effectiveness as a bronchodilating drug or the ability of the drug to stimulate cardiac $\aleph_{2}$-adrenoceptors [24]. The highest risk for adverse cardiovascular events is typically seen during the initiation of $\beta_{2}$-agonist therapy. This is likely due to the development of tolerance to adrenergic stimulation through desensitization and down-regulation that occurs within minutes to hours of drug exposure [24].

The electrolyte disturbances (hypokalemia, hypomagnesemia, and hypophosphotemia) seen with clenbuterol and other $\beta_{2}$ adrenoceptor agonists may play an additional role in cardiac toxicity. Hypokalemia occurs with $\aleph_{2}$-adrenergic stimulation of sodium-potassium ATPase in skeletal muscle that results in an intracellular shift of potassium [10]. In susceptible patients, hypokalemia has been associated with an increased risk for cardiac dysrhythmias [25]. The decrease in serum magnesium and phosphorus are likely due to the concomitant hyperglycemia and intracellular sequestration [10]. It is not known if this patient's electrolyte disturbances contributed to the development of atrial fibrillation.

The treatment of clenbuterol-induced tachydysrhythmias has not been well studied. The use of receptor-targeted therapy with a short-acting $\beta$-antagonist (such as esmolol) is recommended in patients who have no other contraindications. Oral therapy can then be initiated once the acute tachycardia has resolved. Use of a longer-acting $\beta$-antagonist (such as metoprolol or propanolol) may be preferable at that time. With the knowledge that the underlying mechanism is a cellular shift in potassium and not a total body deficit, the resultant hypokalemia should be treated judiciously. Aggressive potassium supplementation may lead to hyperkalemia once the effects of clenbuterol subside.

\section{CONCLUSION}

This is the first case describing the development of supraventricular tachycardia with subsequent atrial fibrillation in a therapeutic 
clenbuterol misadventure. This case exemplifies the common toxicity seen with acute clenbuterol overdose as well as the recognition of its wide-spread use in body-building. The effects of clenbuterol toxicity are similar to other $\beta_{2}$-adrenoceptor agonists, but the unique kinetics of clenbuterol may cause severe and prolonged effects. Treatment is generally supportive by maintenance of vital signs and electrolytes. Considering the large number of body-building enthusiasts, physicians will continue to encounter clenbuterol abuse in their clinical practices.

The authors have no potential financial conflicts of interest to report.

\section{REFERENCES}

1. Goldstein DR, Dobbs T, Krull B, et al. Clenbuterol and anabolic steroids: A previously unreported case of myocardial infarction with normal coronary arteriograms. Southern Med J. 1998;91:780-784.

2. Prather I, Brown D, North P, et al. Clenbuterol: A substitute for anabolic steroids? Med Sci Sports Exerc.

1995;27:1118-1121.

3. Hall H, Sallemark M, Ross S. Clenbuterol, a central ß-adrenoreceptor agonist. Acta Pharmacol et Toxicol. 1980;47: 159-160.

4. Choo J, Horan M, Little R, et al. Anabolic effects of clenbuterol on skeletal muscle are mediated by $B 2$-adrenoreceptor activation. Am J Physiol. 1992;263:E60-E56.

5. Maltin C, Delday M, Watson J, et al. Clenbuterol, a ß2-adrenoreceptor agonist, increases relative muscle strength in orthopaedic patients. Clin Sci. 1993;84:651-654.

6. Spann C, Winter M. Effect of clenbuterol on athletic performance. Ann Pharmacother. Jan 1995;29(1):75-77.

7. Maistro S, Chiesa E, Angeletti R, et al. Beta blockers to prevent clenbuterol poisoning. Lancet. 1995;346:180.

8. Salleras L, Dominguez A, Mata E, et al. Epidemiologic study of an outbreak of clenbuterol poisoning in Catalonia, Spain. Public Health Reports. 1995;

110:338-342.

9. Cortes-Belen E, Suvak M, Benitez J. Clenbuterol overdose in a young female. Clin Toxicol. 1998;36:510-511.

10. Hoffman R, Hoffman R, Freyberg C. Clenbuterol ingestion causing prolonged tachycardia, hypokalemia, and hypophosphatemia with confirmation by quantitative levels. Clin Toxicol. 2001;39:339-344.
11. Chan T. Food-borne clenbuterol may have potential for cardiovascular effects with chronic exposure. Clin Toxicol.

2001;39:345-348.

12. DuRant R, Rickert V, Ashworth C. Use of Multiple Drugs among Adolescents Who Use Anabolic Steroids. NEJM.

1993;328(13):922-926.

13. Leshner A. Anabolic Steroid Use. National Institute of Drug Abuse Research Report Series. 2000; NIH Publication Number 003721:1-8.

14. http://www.steroid.com/CLENBUTEROL.phtml. Available at: http://www.steroid.com/CLENBUTEROL.phtml. Accessed March 3, 2006.

15. Zimmer A. Administration of clenbuterol in man. Single doses, multiple doses, and metabolite samples (unpublished report). 1976. Located at: Submitted to WHO by Boehringer Ingelheim Vetmedica GmbH, Ingelheim, Rhein, Germany.

16. Boehringer Ingelheim Vetmedica I. VENTIPULMIN® Syrup (clenbuterol HCl). St. Joseph, MO 64506-2002. Available at: http://www.boehringer-ingelheim.com. Accessed March 15, 2006.

17. Schnapf B, Santeiro M. Beta-agonist inhaler causing hallucinations. Pediatr Emerg Care. 1994;10(2):87-88.

18. Wiley J, Spiller H, Krenzelok E, et al. Unintentional albuterol ingestion in children. Pediatr Emerg Care.

1994; 10(4):193-196.

19. Sporano V, Grasso L, Esposito M, et al. Clenbuterol residues in non-liver containing meat as a cause of collective food poisoning. Vet Hum Toxicol. Jun 1998;40(3):141-143.

20. Breeden CC, Safirstein B. Albuterol and spacer-induced atrial fibrillation. Chest. Sep 1990;98(3):762-763.

21. Poukkula A, Korhonen U, Huikur H, et al. Theophylline and salbutamol in combination in patients with obstructive pulmonary disease and concurrent heart disease: effect on cardiac arrhythmias. J Intern Med. Oct 1989;226(4):229-234.

22. Lashgari S, Kueck A, Oyelese Y. Atrial fibrillation in pregnancy associated with oral terbutaline therapy. Obstet Gynecol. Apr 2003;101(4):814.

23. Bristow M. Beta-adrenergic receptor blockade in chronic heart failure. Circulation. 2000;101:558-569.

24. Johnson M. The beta-adrenoceptor. Am J Respir Crit Care Med. 1998;158:S146-S153.

25. Nordrehaug J, Johanssen K, Von Der Lippe G. Serum potassium concentration as a risk factor of ventricular arrhythmias early in acute myocardial infarction. Circulation.

1985;71:645-649. 